\title{
Androstenediol Metabolite Measurement
}

National Cancer Institute

\section{Source}

National Cancer Institute. Androstenedio/ Metabolite Measurement. NCI Thesaurus.

Code C74842.

The determination of the amount of androstenediol metabolite present in a sample. 